\title{
Extensive mucocutaneous histiocytic sarcoma raised from an acute b lymphocytic leukemia: a case report at hospital Mexico, Costa Rica
}

\begin{abstract}
Histiocytic sarcomas are rare neoplasms with poor prognosis originating from histiocytic or dendritic cell clones and associated with haematological malignancies such as acute or chronic leukemias and lymphomas. We describe a case of a patient who developed a disseminated and extensive mucocutaneous histiocytic sarcoma during remission.
\end{abstract}

Keywords: transformation, neoplasms, dendritic cell, carboplatin, anthracyclines
Volume 4 Issue 6 - 2017

\author{
Rodríguez-Pineda M,' Chaverri P,' Garcia C,' \\ Landaverde D,' Durán MR,' Tello I,' Carvajal- \\ Cuenca $A,{ }^{2}$ Acon $M,{ }^{2}$ and Mendez $M^{\prime}$ \\ 'Department of Haematology-Oncology, Hospital México, USA \\ ${ }^{2}$ Department of Pathology, Hospital México, USA
}

\begin{abstract}
Correspondence: Miguel A Rodriguez Pineda, HaematologyOncology Department, Hospital México, San José, Costa Rica, USA, Email drrodriguezpineda@gmail.com
\end{abstract}

Received: June II, 2017 | Published: July II, 2017
Abbreviations: TdT, terminal deoxynucleotidyl transferase; CT, computer tomography; HS, histiocytic sarcoma; ALL, acute lymphoblastic leukemia; ICE, ifosfamide, carboplatin and etoposide; BFM, berlin-frankfurt-münster

\section{Introduction}

Histiocytic sarcoma (HS) is a non-Langerhans histiocyte disorder that may have different patterns of clinical presentation depending on the tumor extension and organs involved. It may be related to haematological malignancies such as acute lymphoblastic leukemia or lymphomas. There is no standardized treatment and therefore each case must be discussed in a multidisciplinary team. In terms of systemic therapy there is no "ideal" protocol; however, carboplatin, ifosfamide, etoposide, vincristine, and anthracyclines have been used in schemas designed for patients with aggressive lymphomas. ${ }^{1-4}$ Here, we present the first documented case in Costa Rica of a patient with HS and extensive mucocutaneous involvement developed in a patient with an acute lymphoblastic leukemia (ALL) during chemotherapy treatment.

\section{Case presentation}

A 22 years old young male, farmer, with no relevant past medical history, was admitted into the Haematology Department of our Institution with a history of general malaise, headache and fever starting 5days before consultation. At physical examination he was found to have many axillary and cervical lymphadenopathies smaller than $2 \mathrm{~cm}$ as well as mild splenomegaly. Initial blood work showed a hemoglobin level in $15.2 \mathrm{~g} / \mathrm{L}$, platelets count in $81,000 /$ $\mu \mathrm{l}$ and $98,000 / \mu 1$ leucocytes. Notably, $60 \%$ of lymphoid-like blasts in the peripheral blood smear were seen. The bone marrow biopsy showed a diffuse proliferation of median sized cells with round nuclei, dispersed chromatin, inconspicuous nucleoli and scarce cytoplasm with the following immunohistochemistry: CD3- CD20 ${ }^{+}, \mathrm{CD} 19^{+}$, $\mathrm{CD}_{10}^{+}, \mathrm{CD} 34^{-}, \mathrm{CD} 117^{-}$, positivity for terminal deoxynucleotidyl transferase (Tdt) and negativity for myeloperoxidase. Therefore, a
B-cell lymphoblastic leukemia diagnosis was established. Remission induction therapy was started immediately with a modified version of the standard Berlin-Frankfurt-Münster (BFM) protocol on July 2016, with a good response after the initial steroid therapy $(60 \mathrm{mg} / \mathrm{kg})$.

On day 15 he was sent to the emergency department because of aplasia with an associated Streptococcus pyogenes bacteremia, for which he received antibiotic therapy with sodium penicillin. During the course of the therapy he was re-evaluated for deep vein thrombosis; however, a septic superficial vein thrombosis was diagnosed. Remission was achieved with less than $0.01 \%$ blasts in the bone marrow with three weeks of delay because of the aplasia and infection. During the second phase of the chemotherapy protocol, he was re-admitted for febrile neutropenia in which no infectious agent was documented. During this admission he presented sudden loss of visual acuity with Roth's spots in the fundoscopic exam, compatible with leukemic retinopathy that resolved properly. In the last week of the second cycle he presented multiple lesions on the extremities and dorsal region, they were elevated, umbilicated and growing in size and number. These lesions were classified by dermatology as molluscum contagiosum (Figure 1).

Considering he was in remission, on January 2017 the chemotherapy protocol was continued with high dose methotrexate. Shortly afterwards, a new staging CT scan showed hepatosplenomegaly with signs of infiltration and a lymphadenopathy in the left renal hilum. Skin biopsies taken from the left eyelid skin and the bone marrow showed a diffuse proliferation of malignant cells with large oval nuclei, vesicular chromatin, a single prominent nucleolus and abundant eosinophilic cytoplasm which stained positive for CD4 and CD68 suggestive of histiocytic sarcoma. Both biopsies ruled out an infectious disease and infiltration by other tumors (lymphoma, carcinoma or melanoma) (Figure 2). An ifosfamide, carboplatin and etoposide (ICE) protocol was started considering that the histiocytic sarcoma developed during chemotherapy treatment for ALL and he had already received steroids, anthracyclines, vincristine, cytarabine, methotrexate, cyclophosphamide and L-asparaginase. He advanced 
into febrile neutropenia with soft tissue infection, and due to the extent of his lesions broad antibiotic treatment was initiated with vancomycin, ceftazidime, linezolid and meropenem. Considering the requirement for parenteral nutrition, amphotericin was also started. Unfortunately, the fever did not fall; he progressed into septic shock and died.
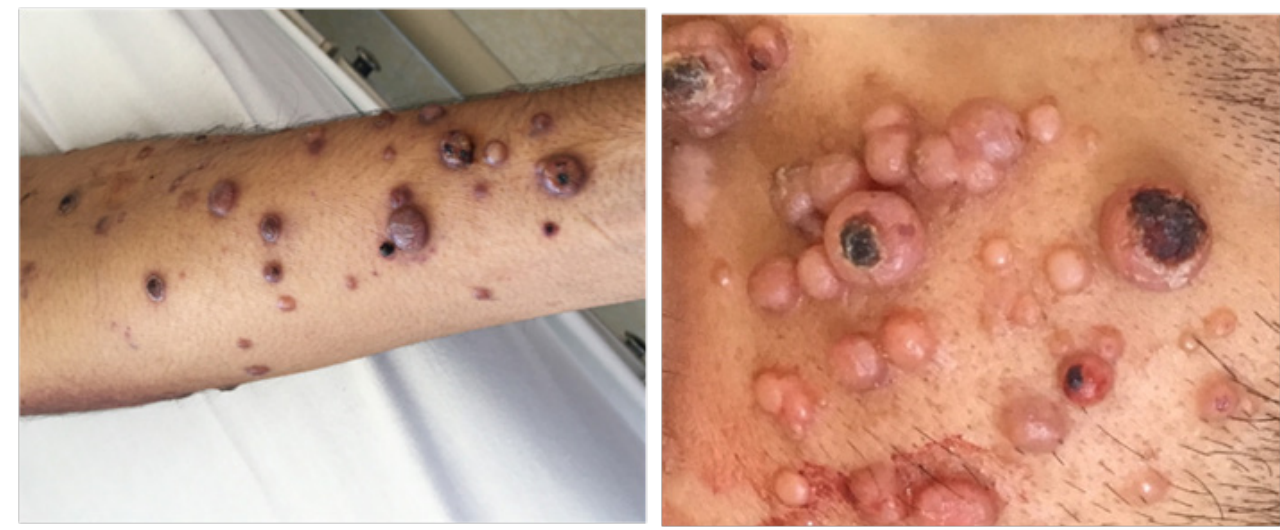

Figure I Elevated, umbilicated skin lesions rapidly developed and were documented in the upper extremities and dorsum.
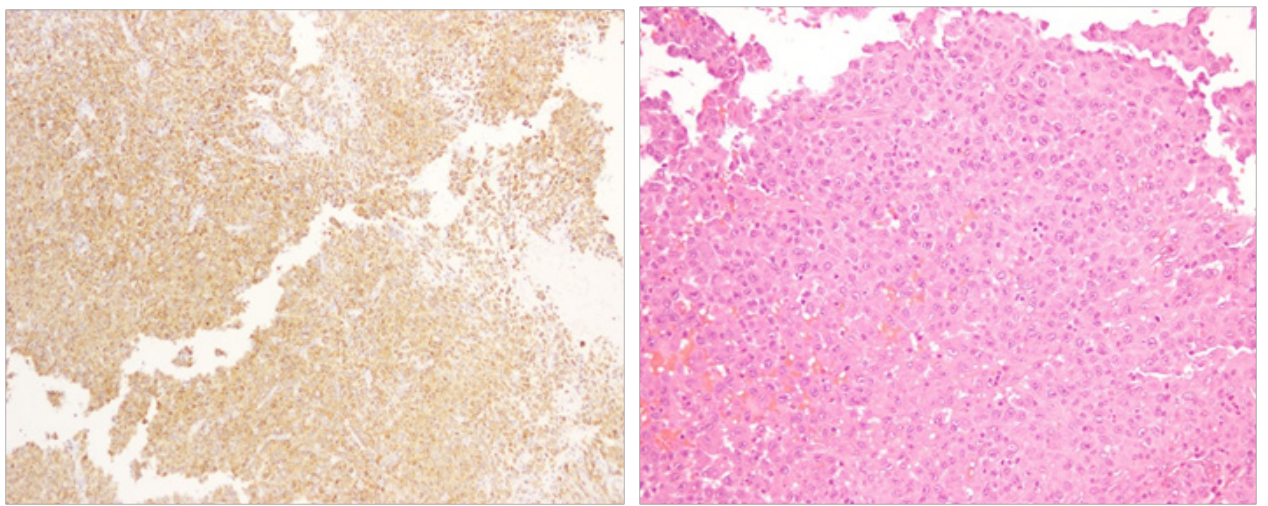

Figure 2 Haematoxylin and Eosin staining of the left eyelid skin biopsy (left panel). Note the diffuse proliferation of malignant cells with large oval nuclei, vesicular chromatin, a single prominent nucleolus and abundant eosinophilic cytoplasm. Hystiocytes stained positive for CD68 (right panel).

\section{Discussion}

HS is an uncommon haematological malignancy categorized as a histiocytic and dendritic cell neoplasm. ${ }^{5}$ It is characterized by the accumulation of macrophages, dendritic cells or monocyte-derived cells in various tissues and organs. ${ }^{6}$ It accounts for less than $1 \%$ of all cases of haemato-lymphoid neoplasms and it has predominance in children and young adult males. ${ }^{7}$ They are described as an entity that may arise post-treatment of acute lymphoid leukemia, either at the end or during the maintenance phase. When they appear after acute lymphoid leukemia, the sarcomas share genetics with leukemia, particularly in the immunoglobulin $\mathrm{H}$ gene or the $\mathrm{T}$ lymphocyte antigen receptor. Unfortunately, in our patient we were unable to screen genetic markers in relapse, which would have further confirmed the nature of the lesions. Nevertheless, HS lesions may be localized to skin or bone which is usually treated with surgery, but in aggressive presentations with invasion to lymph nodes, lung, mediastinum and bone marrow the proliferation index $\mathrm{Ki}-67$ is high. As the patients with extensive skin involvement, these aggressive cases should be treated with chemotherapy and survival is low.

\section{Acknowledgements}

None.

\section{Conflict of interest}

The author declares no conflict of interest.

\section{References}

1. Castro EC, Blazquez C, Boyd J, et al. Clinicopathologic features of lesions following ALL, with a Review of literature. Pediatr Dev Pathol. 2010;13(3):225-237.

2. Ganapule AP, Gupta M, Kokil G, et al. Histiocytic Sarcoma with acute lymphoblastic leukemia a rare association: Case report and literatura review. Indian J Hematol Blood Transfus. 2014;30(Suppl 1):305-308.

3. Alten J, Klapper W, Leuschner I, et al. Secondary histiocytic sarcoma may cause apparent persistence or recurrence of minimal residual disease in childhood acute lymphoblastic leukemia. Pediatr Blood Cancer. 2015;62(9):1656-1660

4. Feldman AL, Minniti C, Santi M, et al. Histiocytic sarcoma after acute lymphoblastic leukaemia: a common clonal origin. Lancet Oncol. 2004;5(4):248-250.

5. Swerdlow SH, Campo E, Harris NL, et al. WHO classification of tumours of haematopoietic and lymphoid tissues. 4th ed. Lyon, France: IARC; 2008.439 p.

6. Emile JF, Abla O, Fraitag S, et al. Revised classification of histiocytoses and neoplasms of the macrophage-dendritic cell lines. Blood. 2016;127(22):2672-2681.

7. Takahashi E, Nakamura S. Histiocytic Sarcoma: an updated literature review based on the 2008 WHO classification. J Clin Exp Hematop. 2013;53(1):1-8. 